\title{
Scanning Compton X-ray microscopy
}

\author{
P. Villanueva-Perez,${ }^{1,2, *}$ @i H. Fleckenstein, ${ }^{1}$ M. Prasciolu, ${ }^{3}$ K. T. Murray ${ }^{3}$ \\ M. Domaracký, ${ }^{1}$ K. Gregorič, ${ }^{4}$ V. Mariani, ${ }^{1}$ L. Gelisio, ${ }^{1}$ M. Kuhn, ${ }^{3}$ J. Hannappel, ${ }^{3}$ \\ O. Yefanov, ${ }^{1}$ N. Ivanov, ${ }^{3}$ I. Sarrou, ${ }^{1}$ D. Pennicard, ${ }^{3}$ J. Becker,${ }^{3}$ M. von Zimmermann, ${ }^{3}$ \\ O. GutowsKI , ${ }^{3}$ A.-C. Dippel, ${ }^{3}$ H. N. Chapman, ${ }^{1,5,6}$ —i] AND S. BAJT ${ }^{3,5}$ (1) \\ ${ }^{1}$ Center for Free-Electron Laser Science, Deutsches Elektronen-Synchrotron DESY, Notkestrasse 85, 22607 Hamburg, Germany \\ ${ }^{2}$ Current address: Synchrotron Radiation Research and NanoLund, Lund University, Box 118, 221 00, Lund, Sweden \\ ${ }^{3}$ Deutsches Elektronen-Synchrotron DESY, Notkestraße 85, 22607 Hamburg, Germany \\ ${ }^{4}$ Biotechnical Faculty, University of Ljubljana, Jamnikarjeva UI. 101, 1000 Ljubljana, Slovenia \\ ${ }^{5}$ The Hamburg Centre for Ultrafast Imaging, Luruper Chaussee 149, 22761 Hamburg, Germany \\ ${ }^{6}$ Department of Physics, Universität Hamburg, Luruper Chaussee 149, 22761 Hamburg, Germany \\ ${ }^{*}$ Corresponding author: pablo.villanueva.perez@cfel.de
}

Received 29 January 2021; revised 17 March 2021; accepted 18 March 2021; posted 22 March 2021 (Doc. ID 421232); published 13 April 2021

\begin{abstract}
X-ray microscopy offers the opportunity to image biological and radiosensitive materials without special sample preparations, bridging optical and electron microscopy capabilities. However, the performance of such microscopes, when imaging radiosensitive samples, is not limited by their intrinsic resolution, but by the radiation damage induced on such samples. Here, we demonstrate a novel, to the best of our knowledge, radio-efficient microscope, scanning Compton X-ray microscopy (SCXM), which uses coherently and incoherently (Compton) scattered photons to minimize the deposited energy per unit of mass for a given imaging signal. We implemented SCXM, using lenses capable of efficiently focusing $60 \mathrm{keV} \mathrm{X-ray} \mathrm{photons} \mathrm{into} \mathrm{the} \mathrm{sub-micrometer}$ scale, and probe its radio-efficient capabilities. SCXM, when implemented in high-energy diffraction-limited storage rings, e.g., European Synchrotron Radiation Facility Extremely Brilliant Source and PETRA IV, will open the opportunity to explore the nanoscale of unstained, unsectioned, and undamaged radiosensitive materials. () 2021 Optical Society of America
\end{abstract}

https://doi.org/10.1364/OL.421232

The biological community has the widespread wish to image three-dimensional (3D) whole cells and organelles intact, unstained, and in their native conditions with nanometer resolution. Various instrumentations, employing different techniques and radiation probes, are being developed to address this wish. For example, conventional optical microscopes use visible light, which limits their achievable resolution to the diffraction limit (hundreds of nanometers) [1]. State-of-the-art super-resolution techniques exploit visible light emitters, such as fluorophores, to overcome this limit [2]. However, most biological samples do not naturally fluoresce; therefore, staining with fluorophores is required. Cryo-electron microscopes have demonstrated the possibility of retrieving atomic resolution of biological molecules, opening a new era in structural biology
[3]. Nonetheless, the limited penetration length of electrons (typically $100 \mathrm{~nm}$ ) does not allow intact whole cells to be imaged and requires sectioning [4]. Another probe to address such a goal are $\mathrm{X}$ rays. The short wavelength $(\lambda)$ and high penetration power of $\mathrm{X}$ rays allow the exploration of unsectioned and unstained samples at a resolution that, in principle, can reach the atomic scale, as demonstrated by crystallography. However, the performance of X-ray microscopes when imaging biological samples or other radiosensitive materials is not only dictated by the optical setup or image reconstruction but by the radiation damage that the sample sustains while being imaged [5]. The most complete structural and functional investigations of the cell utilize all possible probes, using the approaches of correlative microscopy [6]. Improving the sensitivity and resolution of $\mathrm{X}$-ray microscopes beyond current capabilities would greatly improve such studies. Here, we experimentally investigate an approach we previously proposed to achieve that [7].

State-of-the-art X-ray microscopes usually exploit either absorption contrast using soft $\mathrm{X}$ rays in the so-called water window [8,9] or elastic scattering [10-13]. However, the performance of such microscopes when imaging radiosensitive materials is limited by radiation damage [5], requiring techniques that maximize the signal per deposited energy of the probe into each resolution element of the sample. That is, we seek a way to optimize the signal (compared to noise) for a given dose. We recently proposed a novel X-ray imaging technique, scanning Compton X-ray microscopy (SCXM) [7], that enhances the achievable resolution and sensitivity of radiosensitive samples by using all of the scattered and emitted photons from a sample when illuminated with $60 \mathrm{keVX}$-ray photons. At such photon energy, photons are mainly scattered inelastically (Compton). Although Compton scattered photons deposit energy into the sample, the fraction of deposited energy per interaction is so small that it can enhance the achievable sensitivity and resolution. Images have been formed by Compton scattering in the context of X-ray fluorescence mapping [14], but SCXM promises to greatly increase dose efficiency (and, 


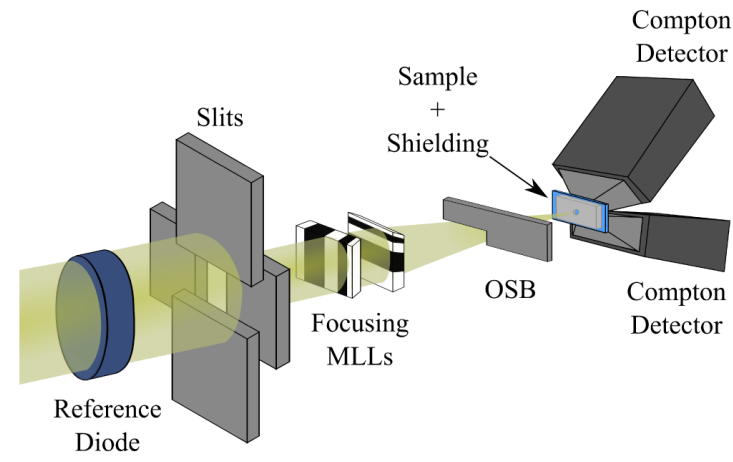

Fig. 1. Schematic of the SCXM setup used at the P07 beamline.

hence, reach higher resolution than previously possible) by optimizing the X-ray photon interaction and experimental setup.

This Letter describes the first proof-of-principle implementation of SCXM. First, we present the SCXM setup and the results of the experiments performed at the P07 beamline [PETRA III, DESY, Germany]. Second, we analyze the reconstructed images and validate the dose-efficient capabilities of SCXM. Finally, we discuss the results in the light of current technical limitations and evaluate further steps necessary to obtain nanometer resolution images of full cells or organelles in natural conditions.

PETRA III is a high-energy storage ring, which operates with a $6 \mathrm{GeV}$ electron beam. The high-energy electron beam enhances the X-ray photon flux at high energies. The highenergy material science beamline (P07, second experimental hutch) provides one of the highest X-ray photon fluxes at $60 \mathrm{keV}$ (approximately $10^{12} \mathrm{ph} / \mathrm{s} / \mathrm{mm}^{2}$ ) [15]. A schematic of the SCXM setup at P07 is shown in Fig. 1.

SCXM is a scanning technique, where the resolution is limited by the focal-spot size [7]. Therefore, lenses capable of focusing high-energy $\mathrm{X}$ rays $(60 \mathrm{keV})$ down to the nanometer scale are needed. Multilayer Laue lenses (MLLs) [16] and multilayer-coated Kirkpatrick-Baez mirrors [17] are the most promising types of X-ray optics that can efficiently focus $\mathrm{X}$ rays to such dimensions. For this experiment, we prepared two off-axis MLLs, each with an aperture of $100 \mu \mathrm{m}$ and focal lengths of $49 \mathrm{~mm}$ and $50 \mathrm{~mm}$, giving a numerical aperture close to $10^{-3}$. These lenses were designed to focus $60 \mathrm{keV} \mathrm{X}$ rays $(\lambda=0.02 \mathrm{~nm})$ to $15 \mathrm{~nm}$ with a depth of focus of $60 \mu \mathrm{m}$. The two lenses were oriented perpendicular to each other to focus in the vertical and horizontal directions and spaced apart from each other by about $1 \mathrm{~mm}$ in the beam direction to ensure stigmatic focusing. Even though the focusing efficiency of these lenses is high, zero-order (non-diffracted) beams cause an undesired background for SCXM. To minimize the contribution from these photons a platinum order-sorting blade (OSB) was inserted between the lenses and the sample. The sample was mounted at the focal plane together with a lead-shielded frame to reduce contributions produced by the scattering of photons from beamline components and the MLLs themselves into the detectors, which would otherwise reduce the Compton contrast.

Compton scattering is distributed over the whole $4 \pi$ solid angle, albeit non-uniformly [18]. The detection of the scattering signal should therefore cover the largest possible solid angle but also consider its angular distribution given that the incident photons are horizontally polarized. Three GaAs 750 k-pixel Lambda detectors were used in this experiment [19], two close to the sample to maximize the Compton signal (see Fig. 1) and one $4 \mathrm{~m}$ downstream. One of the Compton detectors was positioned above the sample plane at a distance of $18 \mathrm{~mm}$ from the center of the detector to the focal spot, covering a solid angle of $2.4 \mathrm{sr}$. The other detector was positioned below the sample plane at a distance of $58 \mathrm{~mm}$ from the center of the detector to the focal spot with a solid angle coverage of $0.55 \mathrm{sr}$. In our original design for SCXM, a beampipe was planned to minimize the background scattering from the air surrounding the sample [7]. Instead, we placed shields over each of the two detector sensors consisting of 3D-printed rectangular plastic pyramids covered with lead foil and small openings on the tips to block all rays impinging on the sensors, except those originating from a small region surrounding the focusing point (see Fig. 1). This greatly reduced background sources and enhanced the measured Compton contrast. The Compton signal was created from the sum of counts in pixels of the two Lambda detectors (allowing very high count rates). A further improvement to the image contrast was made by excluding noisy pixels and pixels near the sensor edge that were shadowed by the shields. The noisy pixels were identified by monitoring the average value of the detector over time and setting a threshold of 10 standard deviations. Any pixel that was above or below those values was automatically masked. After masking those pixels, the remaining signal was flat and uniform across the detector up to variations due to the scattering cross section and solid angle coverage per pixel. The Lambda GaAs detector placed $4 \mathrm{~m}$ downstream was used to monitor the diverging beam transmitted by the sample, to fine tune the alignment of the MLLs, and to estimate the total flux on the sample.

Complementary to the Lambda detectors, three more detectors were used. First, we used a reference diode to normalize the total scattering yield to the incident flux. The diode signal was seen to be well-correlated to the number of photons on the $4 \mathrm{~m}$ downstream Lambda detector. The MMLs, OSB, and samples were brought into coarse alignment with the aid of a high-resolution optical microscope aligned with the $\mathrm{X}$-ray optical axis (on-axis microscope). A scintillator screen with a separate microscope, providing images of the beam behind the sample with $2 \mu \mathrm{m}$ resolution, was used to bring the lenses into their diffracting conditions and to improve the alignment of components. The fine adjustment was then made after slitting down the incident beam (at $60 \mathrm{keV}$ photon energy) to illuminate only the MLLs and monitor the exit pupil in the downstream Lambda detector to ensure the orthogonality of the lenses. This detector was also used to position the OSB to attenuate the undiffracted X-ray photons by the lenses.

The focus distribution was characterized by scanning a lithographically produced gold straight-edge sample and plotting the summed signals of the two Compton detectors as a function of the sample position. Both Compton detectors with their lead-shielding cones were aligned to the tentative focus position. The best results for the horizontal and vertical foci are shown in Figs. 2(a) and 2(b), respectively. To determine the focus size, an error function fit was performed for both axes. The fitted sigma was $250 \pm 30 \mathrm{~nm}$ and $230 \pm 40 \mathrm{~nm}$ for the horizontal and vertical foci, respectively. The retrieved focus was much larger than that based on the MLL design. The main cause of 
(a) Horizontal focus

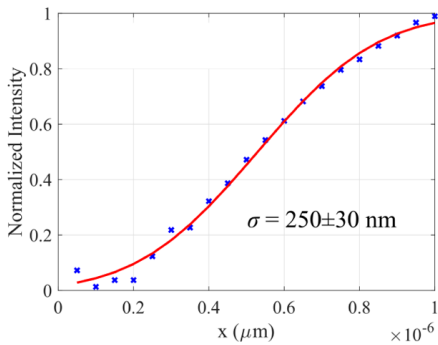

(b) Vertical focus

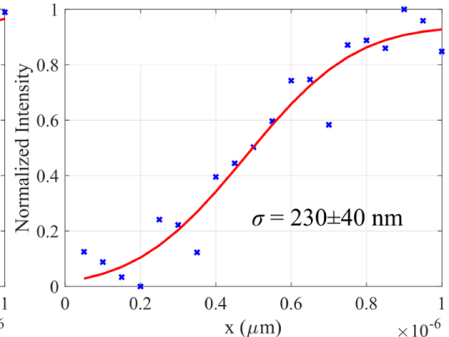

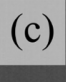

(d)

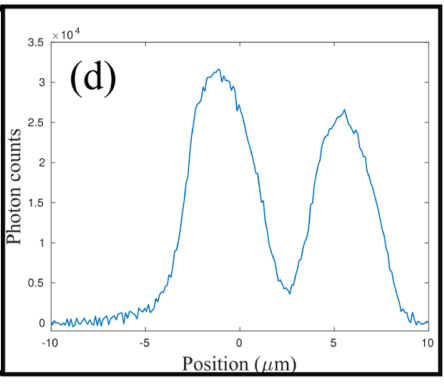

Fig. 2. SCXM scanning results. Focus scan results for the (a) horizontal and (b) vertical focus. The normalized Compton signal is represented by the blue crosses. The red line represents the best fit to an error function. The estimated $\sigma$ for each error function is displayed. (c) Spirulina SCXM image at $60 \mathrm{keV}$ with $100 \mathrm{~nm}$ steps and $100 \mathrm{~ms}$ dwell time. The scale bar corresponds to $10 \mu \mathrm{m}$. (d) Line profile over the red line. The background contrast has been subtracted.

this discrepancy was the transverse coherence of the X-ray beam (or equivalently, the size of the incoherent source imaged by the MLLs).

Arthropira platensis, known as spirulina, was used as the biological sample for testing the dose efficiency of our imaging technique. The most prominent morphological feature of this photosynthetic cyanobacterium is the spiral or helical coiling of its filaments. Several spirulinas were placed on a silicon nitride membrane window. Once in focus, a coarse two-dimensional (2D) scan of a $40 \mu \mathrm{m} \times 40 \mu \mathrm{m}$ area with steps of $100 \mathrm{~nm}$ in the vertical and horizontal directions and a dwell time of $100 \mathrm{~ms}$ was performed. The map resulting from the integrated Compton signal as a function of sample position is depicted in Fig. 2(c). One can clearly see three spirulinas. Although these are $3 \mathrm{D}$ objects, the high depth of focus of $60 \mu \mathrm{m}$ achieved using such a short wavelength (of $0.02 \mathrm{~nm}$ ) is larger than the thickness of the sample. A line profile of the SCXM signal over the red line shown in Fig. 2(c) is displayed in Fig. 2(d). The signal to background maximum contrast of the SCXM was $3.1 \cdot 10^{4}$ photon counts using both Compton detectors. The estimated resolution of Fig. 2(c) is $1.9 \pm 0.7 \mu \mathrm{m}$ by performing an edge fit to one edge. We also performed a Fourier correlation analysis [20] to several edges separated by $1.2 \mu \mathrm{m}$, resulting in a resolution of $1.43 \mu \mathrm{m}$, which is compatible with the edge calculation. These resolution estimations give a lower limit to the retrieved resolution given the $3 \mathrm{D}$ nature of spirulina and the lack of sharp edges, and indeed some band-like features finer than this resolution are visible in Fig. 2(c) (and other images taken).

We also quantitatively compared the signals obtained by SCXM from the spirulina samples with the calculations we used to predict the dose efficiency of this method [7]. We computed signals using our experimental quantities of a flux of $10^{12} \mathrm{ph} / \mathrm{s} / \mathrm{mm}^{2}$ on the MLLs at $60 \mathrm{keV}$ [15] and a dwell time per point of $0.1 \mathrm{~s}$. The spirulina was modeled as a cylinder with a diameter of $8 \mu \mathrm{m}$ with a density of $1.1 \mathrm{~g} / \mathrm{cm}^{3}$ and an average stoichiometry of $\mathrm{H}_{7} \mathrm{C}_{4} \mathrm{NO}_{2}$. The Compton Lambda detectors have an area of $84.46 \mathrm{~mm} \times 28.16 \mathrm{~mm}$ and an efficiency of approximately $30 \%$ at $60 \mathrm{keV}$. Per the design of our apparatus, the detector above (below) the sample was positioned at a distance from the sample of $18 \mathrm{~mm}(58 \mathrm{~mm})$ with an angle of $\theta=0.802 \mathrm{rad}(\theta=0.325 \mathrm{rad})$. The MLLs were considered to have a diffraction efficiency of $80 \%$ at $60 \mathrm{keV}$, and an area of $0.1 \mathrm{~mm} \times 0.1 \mathrm{~mm}$, focusing to an effective focal spot of $\sigma=240 \mathrm{~nm}$ width. Assuming that these parameters and the incoming light is fully horizontally polarized, the expected signal of spirulina in excess of the background is $24,000_{-5000}^{+8000}$ photon counts. The main error contribution is due to the positioning of the detectors. We estimated this error by calculating the difference of counts when the detectors are positioned $5 \mathrm{~mm}$ closer to or further from the sample and assuming there is an error of $1^{\circ}$ in the detector angle with respect to the plane perpendicular to the X-ray beam that contains the sample. This calculation results in an asymmetric error in the photon counts. The agreement between this calculation and the experimental results validates the theory that predicts the dose-efficient capabilities of SCXM [7]. Furthermore, we have evaluated the deposited dose with these parameters. The dose deposited in the spirulina to record the image depicted in Fig. 1(c) is 4.8 MGy. This dose is much higher than $1.1 \mathrm{kGy}$, which is the dose we calculated in our previous work to obtain a resolution of around $1 \mu \mathrm{m}$. In addition to the low total detection efficiency of the setup (of below 6\%), this discrepancy is due to an exposure that is higher than needed for this resolution (the acquisition time was chosen assuming a resolution of $100 \mathrm{~nm})$.

We have presented the first SCXM experiment at $60 \mathrm{keV}$ photon energy by scanning a radiosensitive cyanobacterium, spirulina. We have also demonstrated the capability of SCXM to provide dose-efficient images by confirming the theory proposed in Ref. [7]. The image resolution in this work was limited by spatial coherence of PETRA III. Under these conditions, we obtained a focus spot of $\sigma \approx 240 \mathrm{~nm}$. The enhanced brilliance of high-energy diffraction-limited storage rings (HEDLSRs), such as SPring-8-II [21], Advanced Photon Source upgrade (APS-U) [22], European Syncrotron Radiation Facility Extremely Brilliant Source (ESRF-EBS) [23], and PETRA IV [24], will allow exploration with SCXM of nanometer resolution with high photon fluxes. An estimation of the photon fluxes at $60 \mathrm{keV}$ for HE-DLSRs is presented in Fig. 3, assuming the diffraction-limited phase space $(\lambda / 4 \pi)$ and not the Abbe diffraction limit $(\lambda / 2)[25]$. Furthermore, the detection scheme used here covered only $24 \%$ of the solid angle with $30 \%$ detection efficiency. In the future, we will explore other setups with detectors that cover almost the whole solid angle to capture all of the scattered photons with higher detection efficiency. Such detectors could be based on CdTe or newly suggested schemes using gas detectors [26]. With such a detection 


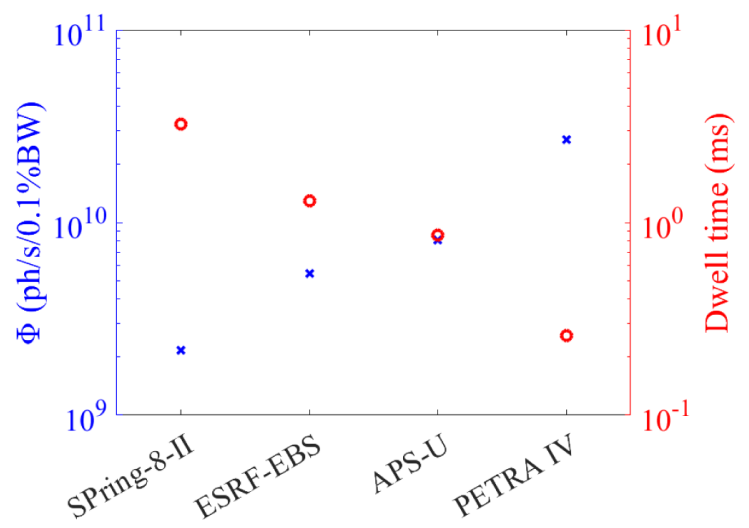

Fig. 3. Coherent flux at $60 \mathrm{keV}$ and SCXM dwell times to retrieve $30 \mathrm{~nm}$ resolution of radiosensitive samples at HE-DLSRs.

scheme and future HE-DLSRs, SCXM will be able to resolve, e.g., $30 \mathrm{~nm}$ DNA features embedded in $5 \mu \mathrm{m}$ of water with millisecond dwell times (see Fig. 3). For this calculation, we have assumed the contrast model presented in Ref. [7], with optics capable of focusing down to $10 \mathrm{~nm}$ with $50 \%$ efficiency, and the diffraction-limited flux provided by HE-DLSRs. Thus, SCXM at HE-DLSRs, together with the proposed improvements, can open up the possibility to obtain nanometer resolution images of unstained and undamaged cells and organelles, offering an approach that bridges the capabilities of optical and electron microscopes.

Funding. Helmholtz-Russian Joint Grant [HRSF-0004 (DESY, Germany), RSF 18-44-06001 (TSU, Russia)]; the Gottfried Wilhelm Leibniz Program of the DFG; Cluster of Excellence "CUI: Advanced Imaging of Matter" of the Deutsche Forschungsgemeinschaft - EXC 2056 (390715994).

Acknowledgment. The authors thank the valuable contributions of Sabrina Bolmer, Heinz Graafsma, Hagen Stawitz, Thomas Keller, Andreas Stierle (DESY), Lars Gumprecht, Dominik Oberthuer (CFEL), Jože Bajuk (Univ. of Ljubljana, Slovenia), and our collaborators from the R\&D Center "Advanced Electronic Technologies" of Tomsk State University, Russia. We acknowledge the use of the FIB dual beam instrument granted by BMBF under grant no. 5K13WC3 (PTDESY).

Disclosures. The authors declare no conflicts of interest.

Data Availability. Data underlying the results presented in this paper are not publicly available at this time but may be obtained from the authors upon reasonable request.

\section{REFERENCES}

1. M. Born and E. Wolf, Principles of Optics (Pergamon, 1999).

2. J. C. Stockert and A. Blazquez-Castro, Fluorescence Microscopy in Life Sciences (Bentham Science, 2017).

3. J. Frank, Nat. Protoc. 12, 209 (2017).

4. S. G. Wolf, L. Houben, and M. Elbaum, Nat. Methods 11, 423 (2014).

5. M. R. Howells, T. Beetz, H. N. Chapman, C. Cui, J. M. Holton, C. J. Jacobsen, J. Kirz, E. Lima, S. Marchesini, H. Miao, D. Sayre, D. A. Shapiro, J. C. H. Spence, and D. Starodub, J. Electron. Spectrosc. Relat. Phenom. 170, 4 (2009).
6. T. Ando, S. P. Bhamidimarri, N. Brending, H. Colin-York, L. Collinson, N. D. Jonge, P. J. de Pablo, E. Debroye, C. Eggeling, C. Franck, M. Fritzsche, H. Gerritsen, B. N. G. Giepmans, K. Grunewald, J. Hofkens, J. P. Hoogenboom, K. P. F. Janssen, R. Kaufmann, J. Klumperman, N. Kurniawan, J. Kusch, N. Liv, V. Parekh, D. B. Peckys, F. Rehfeldt, D. C. Reutens, M. B. J. Roeffaers, T. Salditt, I. A. T. Schaap, U. S. Schwarz, P. Verkade, M. W. Vogel, R. Wagner, M. Winterhalter, H. Yuan, and G. Zifarelli, J. Phys. D 51, 443001 (2018).

7. P. Villanueva-Perez, S. Bajt, and H. N. Chapman, Optica 5, 450 (2018).

8. C. Jacobsen and J. Kirz, Nat. Struct. Biol. 5, 650 (1998).

9. R. Carzaniga, M.-C. Domart, L. M. Collinson, and E. Duke, Protoplasma 251, 449 (2014).

10. H. N. Chapman and K. A. Nugent, Nat. Photonics 4, 833 (2010).

11. C. Holzner, M. Feser, S. Vogt, B. Hornberger, S. B. Baines, and C. Jacobsen, Nat. Phys. 6, 883 (2010).

12. J. Deng, D. J. Vine, S. Chen, Q. Jin, Y. S. Nashed, T. Peterka, S. Vogt, and C. Jacobsen, Sci. Rep. 7, 445 (2017).

13. F. Pfeiffer, Nat. Photonics 12, 9 (2018).

14. C. G. Ryan, D. P. Siddons, R. Kirkham, Z. Y. Li, M. D. de Jonge, D. J. Paterson, A. Kuczewski, D. L. Howard, P. A. Dunn, G. Falkenberg, U. Boesenberg, G. D. Geronimo, L. A. Fisher, A. Halfpenny, M. J. Lintern, E. Lombi, K. A. Dyl, M. Jensen, G. F. Moorhead, J. S. Cleverley, R. M. Hough, B. Godel, S. J. Barnes, S. A. James, K. M. Spiers, M. Alfeld, G. Wellenreuther, Z. Vukmanovic, and S. Borg, J. Phys. Conf. Ser. 499, 012002 (2014).

15. N. Schell, A. King, F. Beckmann, T. Fischer, M. Müller, and A. Schreyer, Materials Science Forum 772, 57 (2013).

16. S. Bajt, M. Prasciolu, H. Fleckenstein, M. Domaracký, H. N. Chapman, A. J. Morgan, O. Yefanov, M. Messerschmidt, Y. Du, K. T. Murray, V. Mariani, M. Kuhn, S. Aplin, K. Pande, P. Villanueva-Perez, K. Stachnik, J. P. Chen, A. Andrejczuk, A. Meents, A. Burkhardt, D. Pennicard, X. Huang, H. Yan, E. Nazaretski, Y. S. Chu, and C. E. Hamm, Light Sci. Appl. 7, 17162 (2018).

17. J. C. da Silva, A. Pacureanu, Y. Yang, S. Bohic, C. Morawe, R. Barrett, and P. Cloetens, Optica 4, 492 (2017).

18. J. H. Hubbell, W. J. Veigele, E. A. Briggs, R. T. Brown, D. T. Cromer, and R. J. Howerton, J. Phys. Chem. Ref. Data 4, 471 (1975).

19. D. Pennicard, S. Smoljanin, B. Struth, H. Hirsemann, A. Fauler, M. Fiederle, O. Tolbanov, A. Zarubin, A. Tyazhev, G. Shelkov, and H. Graafsma, J. Instrum. 9, C12026 (2014).

20. M. Van Heel and M. Schatz, J. Struct. Biol. 151, 250 (2005).

21. H. Tanaka, T. Ishikawa, S. Goto, S. Takano, T. Watanabe, and M. Yabashi, "SPring-8 upgrade project," in 7th International Particle Accelerator Conference (IPAC) (2016).

22. T. E. Fornek, "Advanced photon source upgrade project preliminary design report," APSU-2.01-RPT-002 (2017).

23. P. Raimondi, Synchrotron Radiation News 29(6), 8 (2016).

24. C. G. Schroer, I. Agapov, W. Brefeld, R. Brinkmann, Y. C. Chae, H. Chao, M. Eriksson, J. Keil, X. Nuel Gavalda, R. Roehlsberger, O. H. Seeck, M. Sprung, M. Tischer, R. Wanzenberg, and E. Weckert, J. Synchrotron Radiat. 25, 1277 (2018).

25. M. Born, E. Wolf, A. B. Bhatia, P. C. Clemmow, D. Gabor, A. R. Stokes, A. M. Taylor, P. A. Wayman, and W. L. Wilcock, Principles of Optics: Electromagnetic Theory of Propagation, Interference and Diffraction of Light, 7th ed. (Cambridge University, 1999).

26. A. Saa-Hernandez, D. Gonzalez-Diaz, P. Villanueva-Perez, C. Azevedo, and M. Seoane, "A new imaging technology based on Compton X-ray scattering," https://arxiv.org/abs/2006.01504 (2020). 